\title{
NEW QUANTITATIVE AUTOMATED MODEL TO SIMULATE BACTERIAL DISSEMINATION IN HUMAN TISSUE DURING IRRIGATION OF CONTAMINATED WOUNDS
}

\author{
C. Brochhausen ${ }^{1, \$}$, F. Froschermeier $2, \$$, V. Alt ${ }^{2}$, C. Pfeifer ${ }^{2}$, A. Mayr², I. Weiss ${ }^{2}$, M. Babel $^{1}$, H. Siegmund ${ }^{1}$ and \\ M. Kerschbaum ${ }^{2, *}$ \\ ${ }^{1}$ Institute of Pathology, University Regensburg, Regensburg, Germany \\ ${ }^{2}$ Department of Trauma Surgery, University Medical Centre Regensburg, Regensburg, Germany \\ $\S$ These authors contributed equally to this work
}

\begin{abstract}
This study presents a simple and cost-effective model using microparticles to simulate the bacterial distribution pattern in soft tissue after low- and high-pressure irrigation. Silica coated iron microparticles [comparable diameter $(1 \mu \mathrm{m})$ and weight $(0.8333 \mathrm{pg})$ to Staphylococcus aureus] were applied to the surface of twenty fresh human muscle tissue samples in two amputated lower legs. Particle dissemination into deep tissue layers as an undesired side effect was investigated in four measuring fields as positive control (PC) as well as after performing pulsatile high-pressure (HP, 8 measuring fields) and low-pressure flushing (LP, 8 measuring fields). Five biopsies were taken out of each measuring field to get a total number of 100 biopsies. After histological and digital image processing, the specimens were analysed, and all incomplete sections were excluded. A special detection algorithm was parameterised using the open source bioimage analysis software QuPath. The application of this detection algorithm enabled automated counting and detection of the particles with a sensitivity of $95 \%$ compared to manual counts. Statistical analysis revealed significant differences $(p<0.05)$ in our three different sample groups: $\mathrm{HP}(M=1608, S=302)$, LP $(M=2176, S D=609)$ and PC $(M=4011, S D=686)$.

While both HP and LP flushing techniques are able to reduce the number of bacteria, a higher effectiveness is shown for HP irrigation. Nevertheless, a challenge for the validity of the study is the use of dead tissue and therefore a possible negative influence of high-pressure irrigation on tissue healing and further dispersion of particles cannot be evaluated.
\end{abstract}

Keywords: Soft tissue contamination, microparticles, automated particle count, digital whole slide image processing, jet lavage.

*Address for correspondence: Priv.-Doz. Dr. med. Maximilian Kerschbaum, Department of Trauma Surgery, University Medical Centre Regensburg, Franz-Josef-Strauss Allee 11, 93053 Regensburg, Germany.

Telephone number: +4994194416515 Email: maximilian.kerschbaum@ukr.de

Copyright policy: This article is distributed in accordance with Creative Commons Attribution Licence (http://creativecommons.org/licenses/by-sa/4.0/).

\section{Introduction}

Bacterial soft tissue infections are of increasing interest in clinical research (Gottlieb et al., 2019). This research area concentrates on investigating interactions of pathogens with surrounding tissue and the immune system (Sattler and Kennedy-Lydon, 2017). Wound healing is a multistep process including inflammation, proliferation, and remodelling (Rahim et al., 2017). Infection significantly disturbs this well-orchestrated wound healing system (Zhao et al., 2016). Effective cleaning as well as eradication of infectious agents is crucial to initiate the wound healing processes. An essential step in anti-infectious treatment is the surgical reduction of bacterial load with debridement and careful irrigation (Fry, 2017). When treating and irrigating wounds, the goal is to remove bacteria from the wound and minimise the spread to deeper tissue layers. Therefore, an appropriate method of wound irrigation would result in less contamination and lower particle concentration deep in the tissue. However, little is known about the influence of these physical methods on elimination and dissemination of bacteria to deeper soft tissue levels. To date, there are experimental and clinical studies investigating high- 
(HP) and low-pressure (LP) irrigation in human bone tissue. These studies point to greater tissue damage and intramedullary propagation of bacteria caused by HP irrigation (Bhandari et al., 1998; Bhandari et al., 2015). Negative effects on new bone formation have also been reported (Dirschl et al., 1998). So far, the influence of these methods on the transfer of bacteria to deeper soft tissue levels has only been investigated in animal tissue studies. Some authors concluded that HP irrigation is more effective in removing bacteria from contaminated wounds (Brown et al., 1978; Tabor et al., 1998). Other authors showed that HP compared to LP irrigation causes increased bacterial penetration depth, a higher level of retained bacteria in the wound and, additionally, an increase in cellular death and macroscopic tissue damage (Bhandari et al., 1999; Boyd et al., 2004; Hassinger et al., 2005).

There is a lack of experimental data on the impact of physical forces, such as HP or LP irrigation, on wound contamination in human tissue samples. This deficiency is potentially caused by the lack of models simulating the particle spread in soft tissue infections. An innovative approach to simulate particle dissemination caused by physical forces in wound infections is presented. This method uses microparticles as objects to compare with Staphylococcus aureus in an experimental setup. Utilisation of microparticles, that mimic the physical behaviour of bacteria, enables a simple automated and quantitative analysis of particle distribution following physical wound treatment using HP or LP irrigation.

\section{Materials and Methods}

\section{Specimens and particles}

Two freshly amputated human lower legs were dissected to create measurement fields of $400 \mathrm{~mm}^{2}$ $(20 \mathrm{~mm} \times 20 \mathrm{~mm})$ in each amputated lower extremity. Epidermis, subcutaneous adipose tissue, and muscle fascia were dissected off to expose the muscle tissue (tibialis anterior muscle; Fig. 1a). Further experiments were performed only on those 10 measurement fields in every amputate, exactly where the anterior tibialis muscle was exposed, in order to ensure a high reproducibility of the tests. $50 \mu \mathrm{L}$ of an iron-particlecontaining solution (SiMAG-Silanol, $50 \mathrm{mg} / \mathrm{mL}$, $1.8 \times 1,012$ particles/g, chemicell $\mathrm{GmbH}$, Berlin, Germany) with 10-fold dilution were pipetted onto the surface of each test area (Fig. 1b). Accordingly, approximately 450 million particles were applied within each test field. The diameter of the particles corresponded to that of Staphylococcus aureus, which is about $1 \mu \mathrm{m}$ (Lorian et al., 1985). SiMAG-Silanol particles consist of an iron core and a silica coat with a mean total diameter of $1 \mu \mathrm{m}$ (Häfeli et al., 2005). One of the particles weighs about $0.8333 \mathrm{pg}$, according to manufacturer's specifications. In comparison, a bacterial cell weighs about 1 pg (Davis et al., 1973).

The samples were left untreated for an exposure time of $5 \mathrm{~min} .8$ of the measuring fields (4 in each amputated lower leg) were treated with HP pulsatile lavage (Level 2, Jet Lavage Ocean Jet 200, Bluerock Medical $\mathrm{GmbH}$, Feldafing, Germany) with a pressure of less than 103,421 Pa. LP irrigation with a $50 \mathrm{~mL}$ syringe was performed in 8 measurement fields (4 in each amputated lower leg). $0.9 \% \mathrm{NaCl}$ solution was used as cleaning solution for both procedures. Each measuring field was cleaned for $30 \mathrm{~s}$ at a distance of $50 \mathrm{~mm}$ from the irrigation device to the tissue. An angle of $90^{\circ}$ to the surface was thereby maintained. The remaining 4 fields ( 2 in each amputate) were left untreated to obtain a positive control group. Both amputated lower legs were subsequently frozen to $-4^{\circ}$ C. 5 biopsies were taken from every measuring area using biopsy punches with a length of $20 \mathrm{~mm}$ and a diameter of $2 \mathrm{~mm}$ (Fig. 1c,d). Thus, a total of 5 biopsies were obtained from each of 10 measurement fields from 2 amputated lower extremities, resulting in a total number of 100 tissue samples. The study has been approved by the Ethics Committee of the University of Regensburg in accordance with the Helsinki Declaration (ref.-number 18-1160-101).

\section{Histology and electron microscopy evaluation}

Punch biopsies from each measuring field were fixed in phosphate-buffered formaldehyde $(4 \%, \mathrm{pH} 7)$

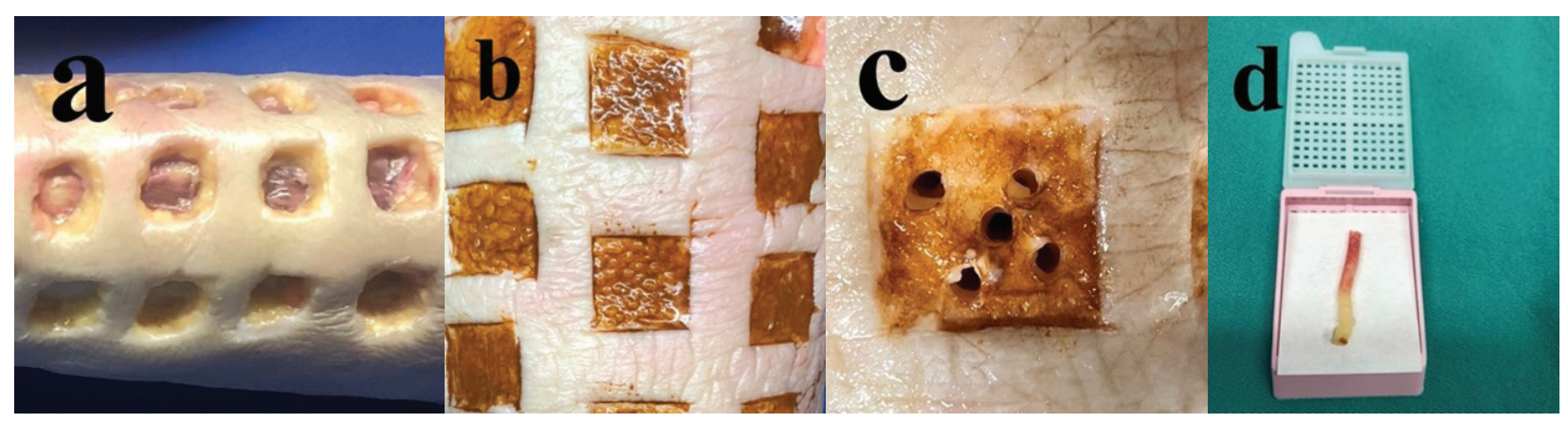

Fig. 1. Preparation of two freshly amputated human lower legs. (a) Measuring fields with a size of $400 \mathrm{~mm}^{2}$ $(20 \mathrm{~mm} \times 20 \mathrm{~mm})$ were prepared in fresh human muscle tissue samples from an amputated lower leg. (b) Measuring fields with applied iron particle solution. (c,d) Five biopsies were taken from each area. 
dehydrated and paraffin wax embedded according to standardised and automated methods (Leica ASP 300S dehydration system, Leica Biosystems; Thermo HistoStar $^{\mathrm{TM}}$, Thermo Scientific). Paraffin wax sections of $4 \mu \mathrm{m}$ thickness were cut (Microm HM 355S, Thermo Scientific ${ }^{\mathrm{TM}}$ ) and stained with haematoxylin and eosin (H\&E), elastica-van Gieson (EvG) and Berlin blue in a standardised and automated manner (Sakura Tissue-Tek Prisma ${ }^{\circledR}$, Sakura Finetek, Alphen aan den Rijn, the Netherlands). Digital images of the slides were capturerd (Pannoramic Digital Slide Scanner, 3DHISTECH, Budapest, Hungary), using a $\times 40$ objective. Resulting whole slide images were reviewed using a digital light microscope (CaseViewer, 3DHISTECH, Budapest, Hungary). Those specimens that were incompletely sectioned were excluded from further analysis [analysed samples: HP: $n=17$; LP: $n=10$; positive control (PC): $n=7]$.

For electron microscopy analysis, formalin fixed tissue samples were post-fixed with cacodylatebuffered glutaraldehyde $(4 \%, \mathrm{pH} 7.2)$ for at least $72 \mathrm{~h}$ at $4{ }^{\circ} \mathrm{C}$ and then cut into smaller samples $\left(2 \mathrm{~mm}^{3}\right)$ using a razor blade. For the embedding process [postfixation with cacodylate-buffered osmium tetroxide (1 \%, pH 7.2), dehydration in graded ethanols, infiltration with Epon (EMbed 812)] the LYNX microscopy tissue processor (Reichert-Jung, Wetzlar, Germany) was used. Semi-thin-sections and ultra-thin sections $(80 \mathrm{~nm})$ were cut using the Reichert Ultracut S Microtome (Leica-Reichert, Wetzlar, Germany). Ultra-thin-sections were stained using aqueous $2 \%$ uranyl acetate and $2 \%$ lead citrate solution for $10 \mathrm{~min}$ each. Electron-microscopy analysis was performed using an EFTEM LEO 912AB electron-microscope (Zeiss). Iron nanoparticles were detected by electron energy loss spectroscopy (EELS) and the distribution was shown by electron spectroscopic imaging.

\section{Quantitative evaluation of particle distribution}

An algorithm for automated quantitative evaluation of the scans was parameterised from the bioimage analysis software QuPath (developed at the University of Edinburgh, UK). This open-source program (Web ref. 1) enables automated digital analysis of whole slide images (Bankhead et al., 2017). In the software, there is a preset function for automated detection and counting of cells. Depending on which cells are to be recognised by the program, the detection criteria can be adjusted according to special characteristics such as size and staining of the target cells. The detection parameters were modified so that the used particles could be recognised by the algorithm. For this purpose, a script that detects the different colouration of the iron microparticles compared to the background was parameterised. In addition, the small size of the particles compared to the large cells in the tissue background is an important distinguishing feature that was taken into account when designing the script. The script was used to automatically analyse the areas previously assigned manually to specific tissue types. The quality of this automated particle detection was validated. 30 high-magnification fields from the centre of the preparations were randomly selected for this purpose, by the software. Automated and manual particle counts were performed on these 30 highmagnification fields. Sensitivity of the automated quantitative evaluation was calculated in comparison to manual counts. To reduce the risk of errors due to dissemination of particles from the surface to deeper areas during biopsy collection, the margins of the histological samples were excluded from quantitative analysis. The superficial particle layer and connective tissue below the fascia were also excluded from quantitative analysis so that only particles in muscle tissue would be analysed. In addition, overlapping, blurred or non-contagious areas were excluded. The resulting particle distribution data were exported. The number of detections was offset against the area. Resulting data were evaluated using SPSS. A oneway analysis of variance (ANOVA) was conducted to assess the effects of irrigation methods on the particle concentration (measured as particle number per $\mathrm{mm}^{2}$ ). Additionally, statistical analysis was conducted for three groups HP, LP and PC resulting in Games-Howell post-hoc analysis.

\section{Results}

\section{Histological detection of particles}

Particles were detected in every staining type by conventional light microscopy, due to their original brown colour. The contrast between particles and background was best following Berlin-blue staining, compared to H\&E and EvG stainings (Fig. 2). In all analysed biopsies, particles were detected on the surface of the specimen and in deeper tissue levels. The particles were visualised by both conventional microscopy and following whole slide imaging. Furthermore, particles were also identified by electron microscopy. In this context, the iron core of the particles was clearly characterised by electron energy loss spectroscopy (Fig. 3).

\section{Quantitative evaluation of particle distribution}

The software allowed the detection of iron particles in Berlin-blue-stained slices due to their different size and contrasting colouration compared to the background. The distribution pattern of particles could only be assessed, using at least a $20 \times$ objective lens for conventional microscopy, due to the small particle size. After applying the detection script, particles were marked by $100 \mu \mathrm{m}$ diameter circles (Fig. 4). The particles, and even clusters, could thereby be displayed in the whole slide image overview for inspection and comparison by conventional histological analyses (Fig. 5). Example whole slide images with particles marked in red from each of the irrigation groups and the control group were included (Fig. 6). It would be difficult to detect any 
difference in particle concentration or distribution pattern, between the different irrigation groups, from these images by visual analysis. However, automated counting enabled quantitative analysis despite this challenge.

Validation of the automated particle count was confirmed by comparing the results with those obtained by manual counting, in 30 predefined high-magnification fields. The mean count in these 30 areas was $43.5 \pm 13.2$ particles by manual evaluation, compared to $44.8 \pm 13.2$ particles by automated analysis. This results in a sensitivity of $95 \%$, comparing the automated algorithm count to the manual procedure. A one-way ANOVA was conducted to assess the effects of irrigation methods on the particle concentration deep in the tissue (measured as particle number per $\mathrm{mm}^{2}$ ). There were 3 different sample groups: $\mathrm{HP}(M=1608, S D=302)$, $\operatorname{LP}(M=2176, S D=609)$ and PC $(M=4011, S D=686)$. There were no outliers, according to inspection using

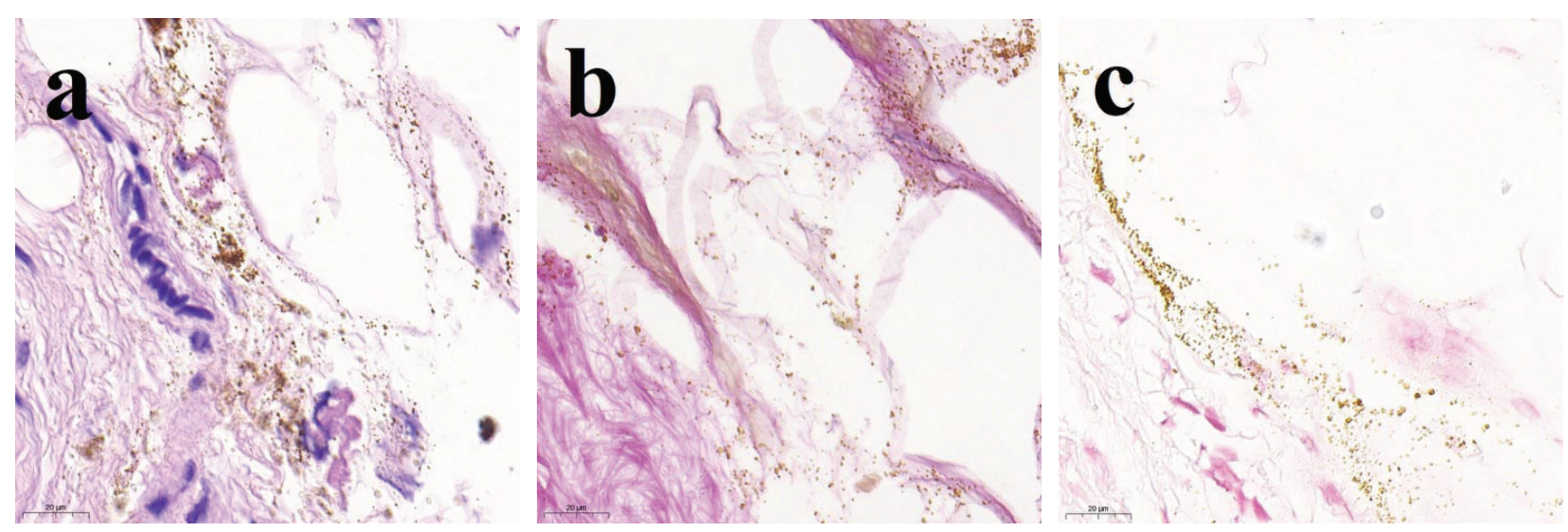

Fig. 2. Histological evaluation of staining types by light microscopy. Particles were visible by light microscopy. Microparticles were detectable as regular brown particles by light microscopy in every staining type. Contrast between particles and background was best following staining using Berlin blue (c), compared to H\&E (a) and EvG (b).

Fig. 3. Electron-microscopy analysis and Fe nanoparticles identified by EELS. (a,b) Red labelling of microparticles. (c) Fe nanoparticles identified by EELS. The distribution was shown by electron spectroscopic imaging.
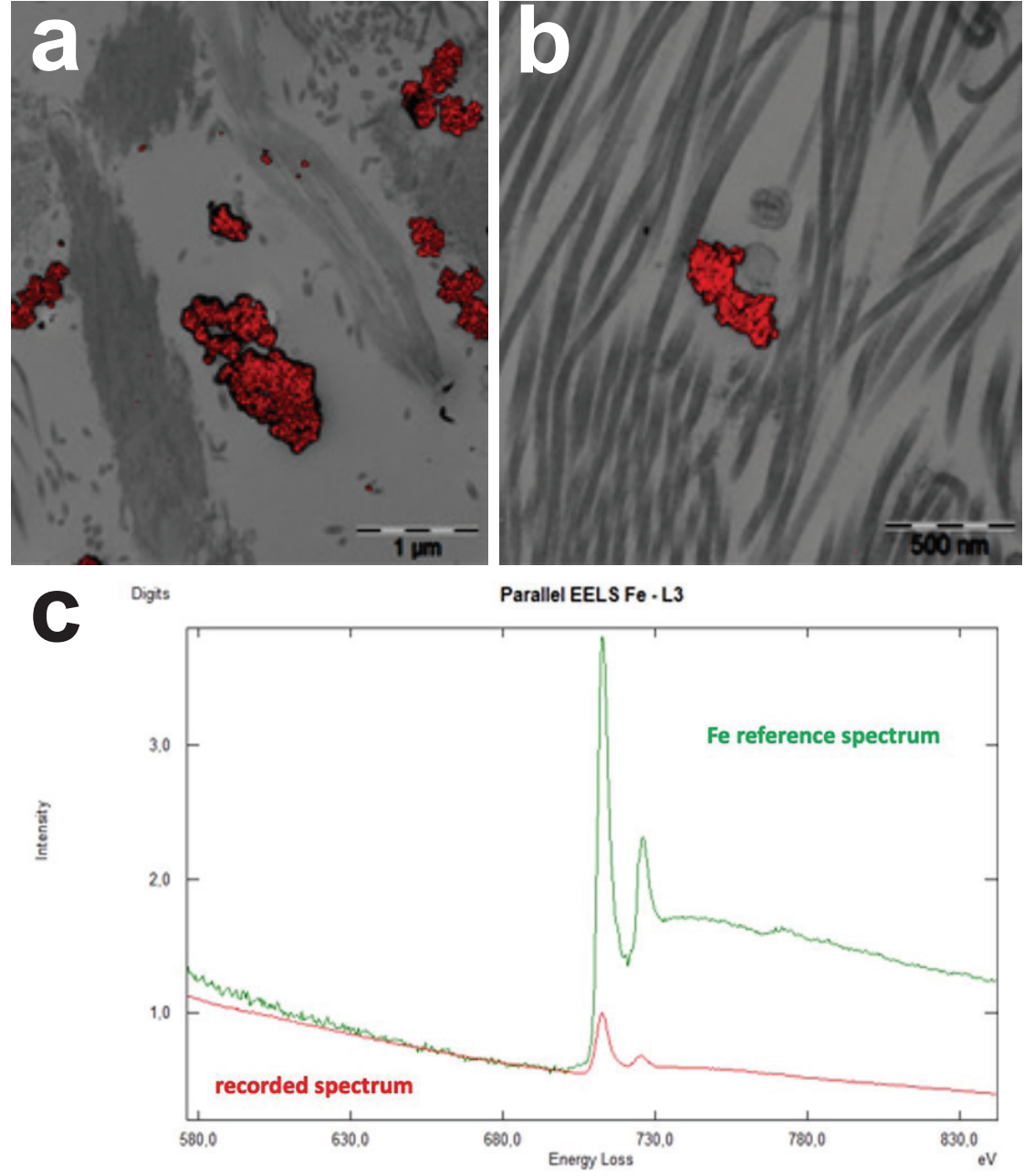

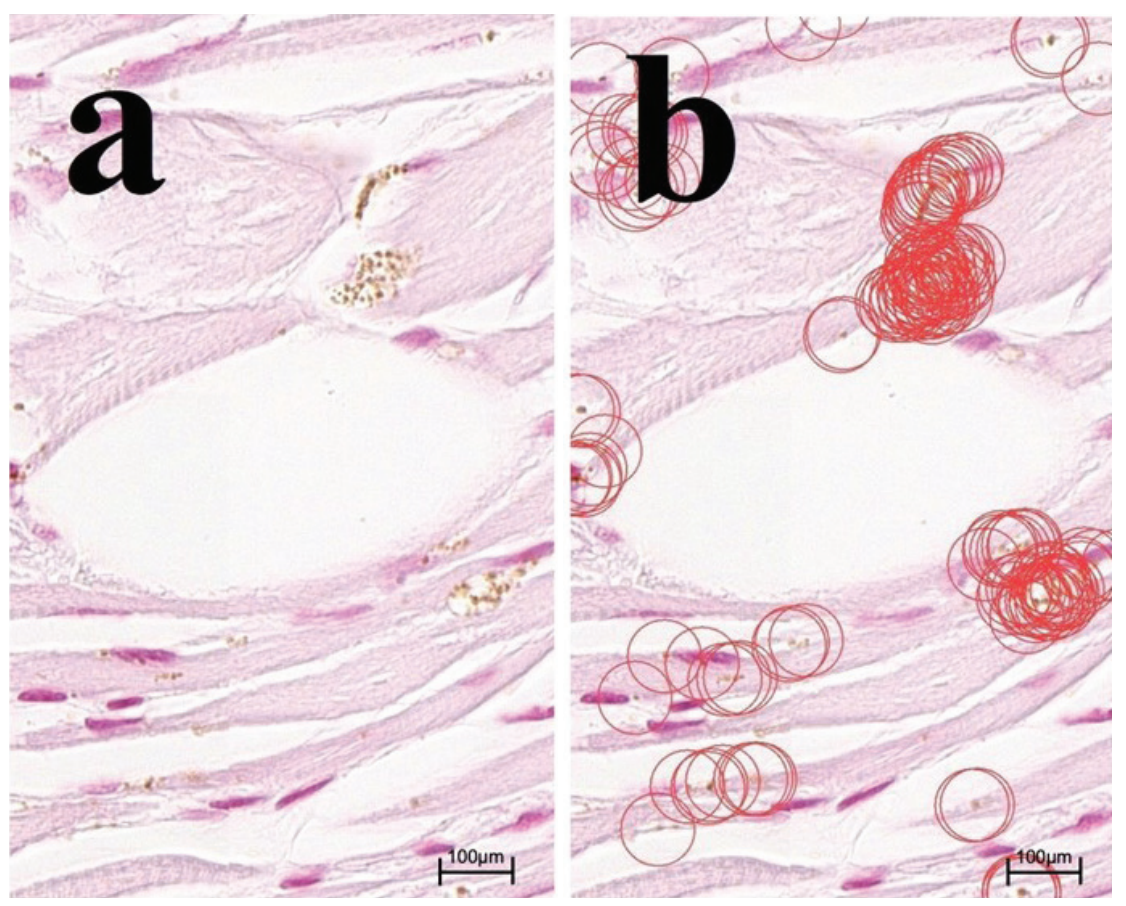

Fig. 4. Marking the particles after applying the script. (a) Running the script allowed detection of iron particles in Berlin blue stained slices due to their different size and colouration compared to the background. (b) Microparticles are marked by red circles with a diameter of $100 \mu \mathrm{m}$.

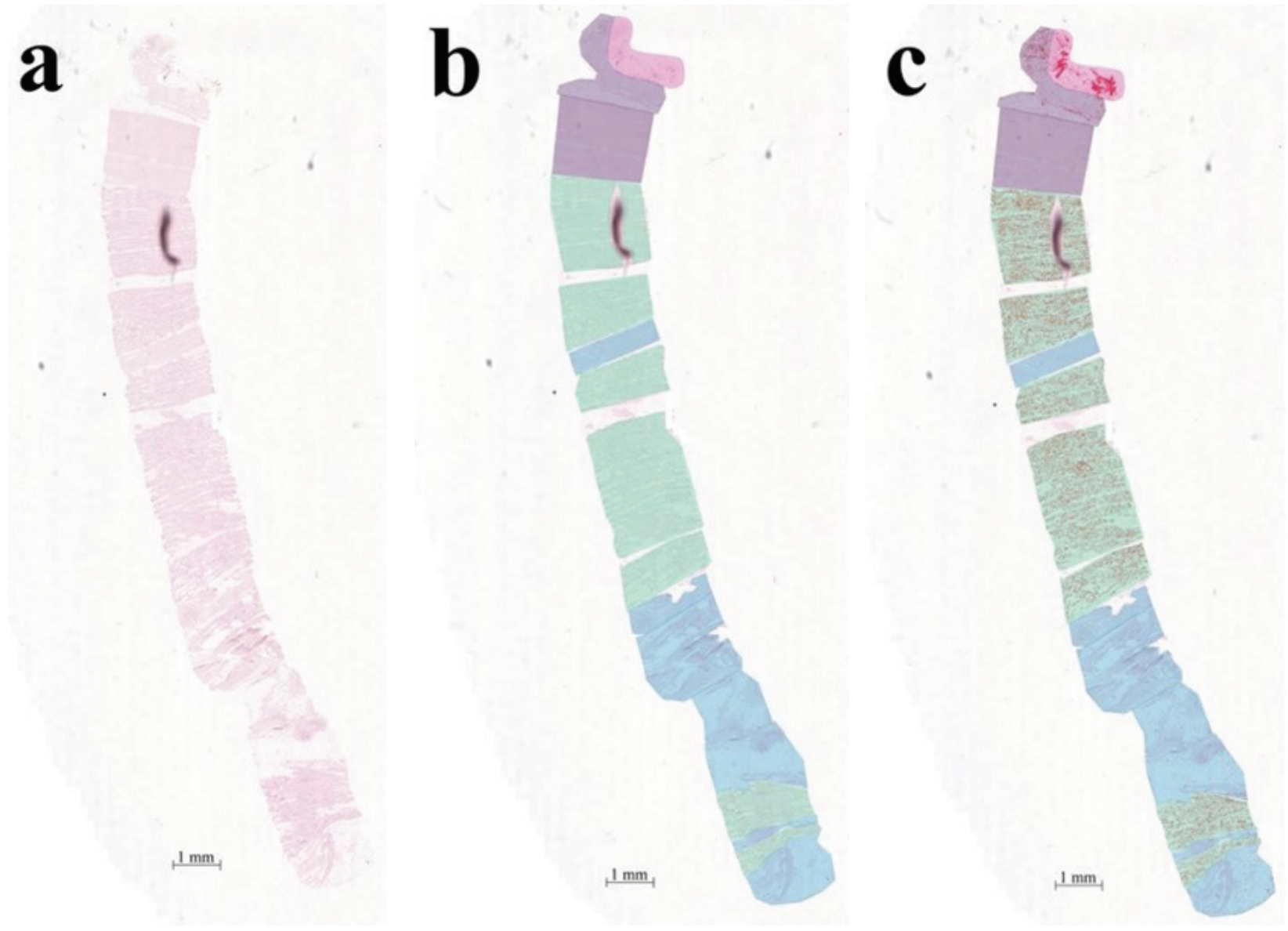

Fig. 5. Whole slide images with tissue-specific regions. (a) Original whole slide images sample. (b) The tissue-specific regions (superficial particle layer, subcutis, fascia, muscle, subfascial connecting tissue) are marked in different colours. (c) The particles are displayed in red in the overview after applying the automated particle detection script. Tissue sections that had detached from the tissue composite at the edge of the biopsies were excluded from the analysis. 
a box-plot. Data were normally distributed for each group (Shapiro-Wilk test, $p>0.05$ ). Homogeneity of variance was not given (Levene's test, $p<0.05$ ). The level of depression for the different levels of physical activity was statistically significant, Welch's $F(2,31)=588,5 ; p<0.001$. Games-Howell post-hoc analysis revealed a significant difference $(p<0.05)$ between particle concentration in all groups. Mean level of particle concentration increased from HP to LP $[+567,9,95 \%-C I(16,3233 ; 1119,5)]$, from HP to PC [+ 2403, $95 \%-C I(1607,9 ; 3198)]$, and from LP to PC [+ 1835, 95 \%-CI $(972,8 ; 2697,2)]$ (Fig. 7).

\section{Discussion}

The present study demonstrated a simple and costeffective model for simulating bacterial dissemination in muscle tissue, following HP and LP flushing using iron particles. These microparticles were detectable by light microscopy as well as electron microscopy. The particles were easily distinguishable, following Berlin blue staining, whereas the visualisation of bacteria would need complex special histological staining. Automated counting enabled precise evaluation of particle distribution in large sample

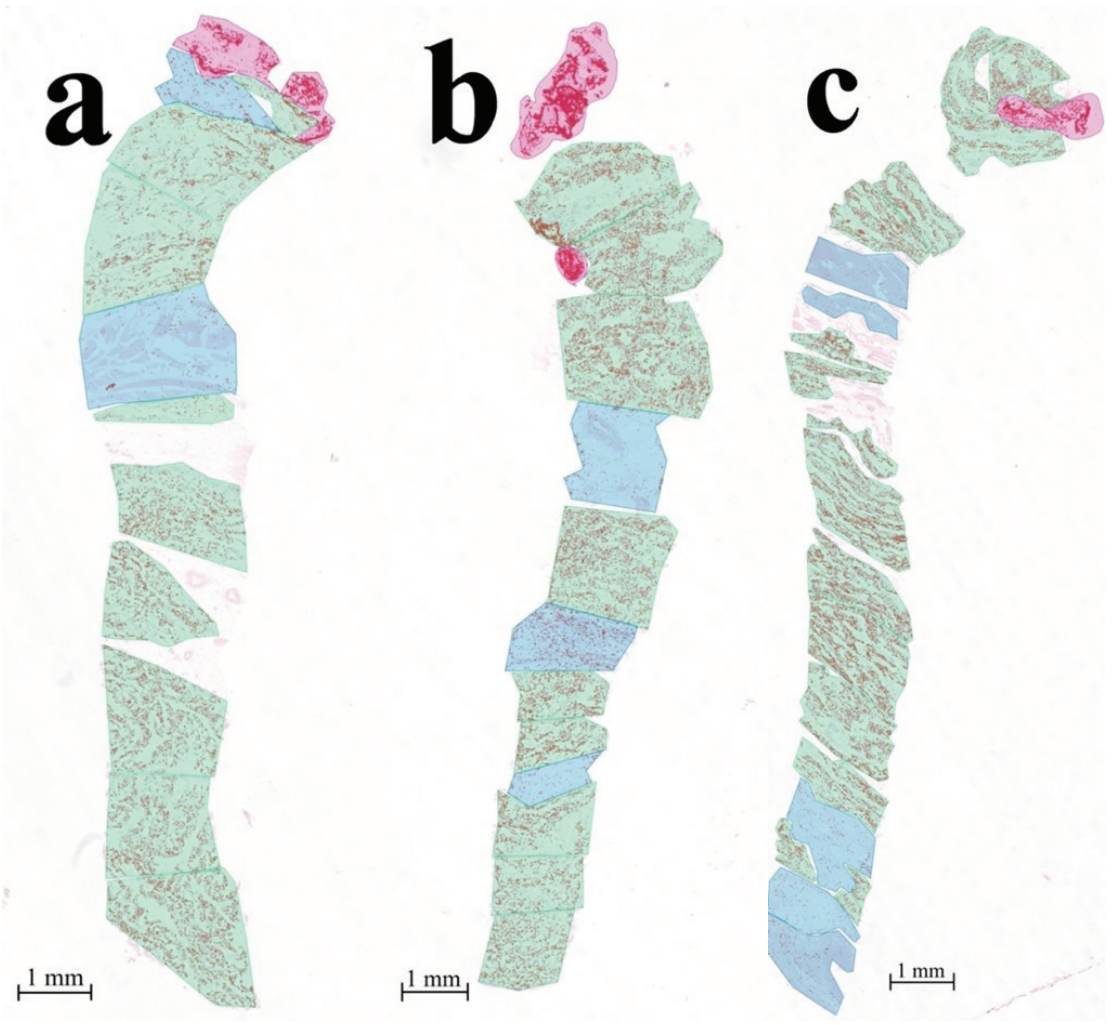

Fig. 6. Whole slide image overviews punch biopsies. (a) After HP irrigation. (b) After LP irrigation. (c) Positive control samples.

Fig. 7. Comparison of HP and LP group with the PC samples of particle density after automated particle count. A script for automated quantitative evaluation of the scans was employed with the help of the bioimage analysis software QuPath. Particle size, and colouration compared to the background are detected. The PC samples showed significantly higher particle density compared to both HP and LP. The HP group also showed significantly fewer particles than the LP group. ${ }^{*} p<0.05$; error bars: SD.

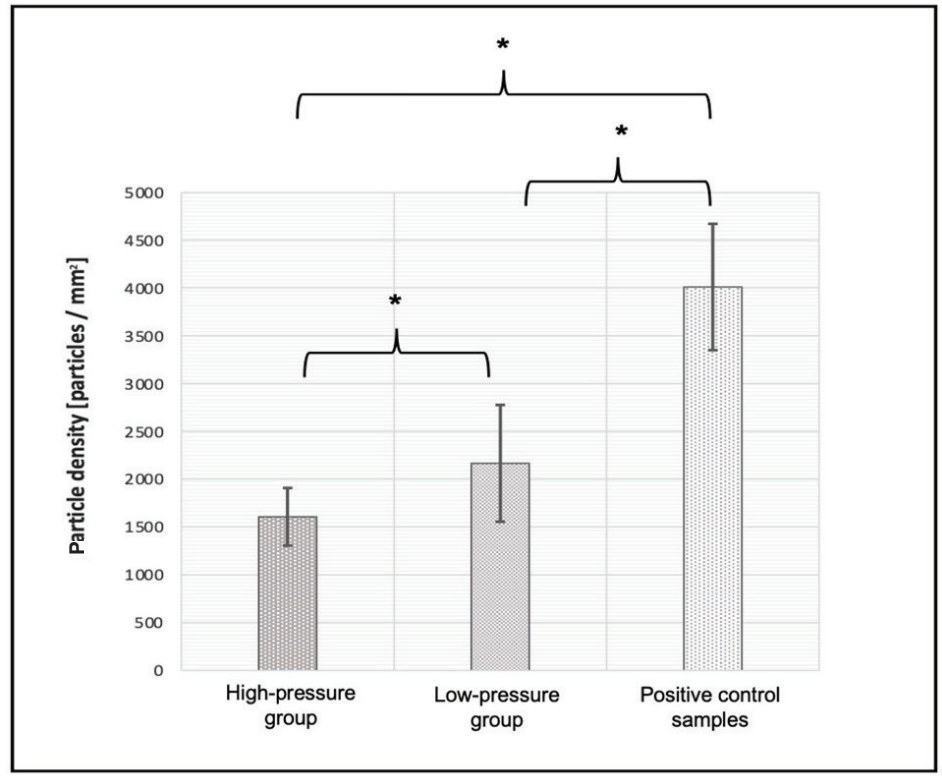


numbers. Since the present model was based on freshly amputated human lower legs, the tissue characteristics represented a physiological situation. Nevertheless, a major challenge for the validity of the study samples is that they were taken from dead tissue. This means that drawing conclusions concerning dissemination through blood vessels and other structures in living tissue remains difficult.

Applying iron microparticles to simulate bacteria offers new possibilities in medical research and costs are reasonable [about $40 \mathrm{US} \$(30 €)$ per $1 \mathrm{~mL}$ containing $50 \mathrm{mg}$ of SiMAG Silanol particles]. Another advantage of this procedure is that experiments such as this can be carried out in a normal laboratory, without special precautions e.g. biohazard qualifications for use of (inactivated) bacteria are not required.

Automated analysis enables objective and unbiased evaluation of histopathological questions (Colling et al., 2019; Huss and Coupland, 2020). The results can be objective, applying a predefined script to each section. Counting the number of particles in a conglomerate, by conventional light microscopy, remains challenging. The algorithm is even capable of detecting single particles in clusters by splitting the shape of a cluster. The automated particle count showed a high sensitivity for particle detection. This automated procedure may also be useful for evaluation of large-scale analysis of samples. Moreover, the present study is the first study on this topic to use fresh human muscle tissue samples (amputated lower legs), whereas previous studies were performed on animal tissue (Boyd et al., 2004; Hassinger et al., 2005). Due to the use of human tissue, the validity of the results is significantly higher than in the previous studies and can therefore be more easily transferred to clinical questions. Previous studies, assessing the effectiveness of different flushing methods on bacterial clearance, have provided contradictory results. While some authors concluded that HP irrigation is more effective in removing bacteria from contaminated wounds (Brown et al., 1978; Tabor et al., 1998), other studies showed that the amount of retained bacteria is higher after HP than LP irrigation methods (Bhandari et al., 1999; Boyd et al., 2004; Hassinger et al., 2005). In the current study, lower particle concentrations were detected in deeper tissue layers after performing HP lavage than following LP lavage. The results of the present study on bacterial clearance from human tissue suggested that pulsating HP irrigation was more effective than the LP method. Nevertheless, the present results also indicated that both methods could significantly reduce the bacterial load (compared to PC). This was consistent with the limited clinical data available on this topic.

In a large-scale randomised controlled clinical trial, it was found that there were no significant differences between HP and LP irrigation for contaminated wounds, in terms of reoperation rate outcomes
(FLOW investigators et al., 2015). The methodological heterogeneity of experimental work on this topic makes it difficult to draw valid conclusions in this area. The present simulation model could help investigations of novel irrigation methods in a costeffective and simple way, with respect to cleaning efficacy and bacterial dissemination within human tissue.

Some limitations of the presented model need to be discussed. Bacteria have bioadhesive properties to tissue composites and use quorum sensing to communicate with each other (Kai 2018). These properties may influence the kinematic characteristics of bacteria and cannot be imitated by lifeless particles. Bacterial cells can even respond to environmental influences (e.g. chemical, thermal or mechanical). One example is the ability of bacteria to use a flagellum for a certain degree of mobility in tissues when responding to mechanical stimuli (Persat et al., 2015). This type of self-mobility cannot be represented by the present particle model. The microbiome in the wound itself influences contaminating bacterial species and thus also has an impact on wound healing, which cannot be simulated (Scales and Huffnagle, 2013). Furthermore, the present model cannot simulate any tissue changes caused by an inflammatory process. At this point, further studies are needed to compare particle behaviour with the behaviour of living bacteria in tissue. Results might be influenced by the fact that only biopsies and not the whole measuring area can be investigated using this model. In addition, dissemination of particles through punch biopsy sampling is possible. An additional limitation of the model is the fact that no information is available on whether and to what extent the iron microparticles cause damage when penetrating the tissue. To limit this source of error, the biopsies were taken from the frozen amputated lower legs and, in addition, the marginal areas of the punch cylinders were excluded from the quantitative analysis.

\section{Conclusions}

The presented method is simple and cost effective and can reproducibly simulate particle dissemination in contaminated soft tissue after HP or LP irrigation. This model represents a promising approach for investigating the influence of different cleaning methods on bacterial transmission in open human wounds. The study showed a higher effectiveness in particle elimination of HP irrigation compared to LP flushing. Both methods were more effective in reducing the amount of bacteria than the positive control. Nevertheless, a challenge for the validity of the study is the use of dead tissue. Therefore, a possible negative influence of HP irrigation on tissue healing and further dispersion of particles cannot be evaluated. 


\section{Acknowledgements}

The department of trauma surgery received funding of Manfred-Roth-Stiftung for their research on soft tissue infections.

\section{Author contributions statement}

Christoph Brochhausen, Christian Pfeifer, Maximilian Kerschbaum and Volker Alt conceived experiments. Heiko Siegmund, Isabella Weiß and Agnes Mayr carried out experiments. Franziska Froschermeier analysed data. All authors were involved in writing the paper and finally approved the submitted version.

\section{Conflict of interest statement}

The authors, whose names are listed on the title page, certify that they have NO affiliations with or involvement in any organisation or entity with any financial interest (such as honoraria, educational grants, participation in speakers' bureaus, membership, employment, consultancies, stock ownership, or other equity interest, and expert testimony or patent-licensing arrangements), or nonfinancial interest (such as personal or professional relationships, affiliations, knowledge or beliefs) in the subject matter or materials discussed in this manuscript.

\section{References}

Bankhead P, Loughrey MB, Fernández JA, Dombrowski Y, McArt DG, Dunne PD, McQUaid S, Gray RT, Murray LJ, Coleman HG, James JA, SaltoTellez M, Hamilton PW (2017) QuPath: open source software for digital pathology image analysis. Sci Rep 7: 16878. DOI: 10.1038/s41598-017-17204-5.

Bhandari M, Adili A, Lachowski RJ (1998) High pressure pulsatile lavage of contaminated human tibiae: an in vitro study. J Orthop Trauma 12: 479-484.

Bhandari M, Jeray KJ, Petrisor BA, Devereaux PJ, Heels-Ansdell D, Schemitsch EH, Anglen J, Della Rocca GJ, Jones C, Kreder H, Liew S, McKay P, Papp S, Sancheti, Sprague PS, Stone TB, Sun X, Tanner SL, Tornetta P, Tufescu T, Walter S, Guyatt GH (2015) A trial of wound irrigation in the initial management of open fracture wounds. N Engl J Med 373: 2629-2641.

Bhandari M, Schemitsch EH, Adili A, Lachowski RJ, Shaughnessy SG (1999) High and low pressure pulsatile lavage of contaminated tibial fractures: an in vitro study of bacterial adherence and bone damage. J Orthop Trauma 13: 526-533.

Boyd JI, Wongworawat MD (2004) High-pressure pulsatile lavage causes soft tissue damage. Clin Orthop Relat Res 427: 13-17.
Brown LL, Shelton HT, Bornside GH, Cohn I. Evaluation of wound irrigation by pulsatile jet and conventional methods (1978) Ann Surg 187: 170-173.

Colling R, Pitman H, Oien K, Rajpoot N, Macklin P, CM-Path Al in Histopathology Working Group, Snead D, Sackville T, Verril C (2019) Artificial intelligence in digital pathology: a roadmap to routine use in clinical practice. J Pathol 249: 143-150.

Davis BD, Dulbecco R, Eisen HN, Ginsberg H (1973) Bacterial physiology: microbiology (2nd edn). Harper and Row: Maryland 96-97.

Dirschl DR, Duff GP, Dahners LE, Edin M, Rahn BA, Miclau T (1998) High pressure pulsatile lavage irrigation of intraarticular fractures: effects on fracture healing. J Orthop Trauma 12: 460-463

FLOW Investigators, Bhandari M, Jeray KJ, Petrisor BA, Devereaux PJ, Heels-Ansdell D, Schemitsch EH, Anglen J, Della Rocca GJ, Jones C, Kreder H, Liew S, McKay P, Papp S, Sancheti P, Prague S, Stone TB, Sun X, Tanner SL, Tornetta P $3^{\text {rd }}$, Tufescu T, Walter S, Guyatt GH, (2015) A trial of wound irrigation in the initial management of open fracture wounds. $\mathrm{N}$ Engl J Med 373: 2629-2641.

Fry DE (2017) Pressure irrigation of surgical incisions and traumatic wounds. Surg Infect (Larchmt) 18: 424-430.

Gottlieb M, DeMott JM, Hallock M, Peksa GD (2019) Systemic antibiotics for the treatment of skin and soft tissue abscesses: a systematic review and meta-analysis. Ann Emerg Med 73: 8-16.

Hassinger SM, Harding G, Wongworawat MD (2005) High-pressure pulsatile lavage propagates bacteria into soft tissue. Clin Orthop Relat Res 439: 27-31.

Häfeli UO, Lobedann MA, Steingroewer J, Moore LR, Riffle J (2005) Optical method for measurement of magnetophoretic mobility of individual magnetic microspheres in defined magnetic field. J Magn Magn Mater 293: 224-239.

Huss R, Coupland SE (2020) Software-assisted decision support in digital histopathology. J Pathol 250: 685-692.

Kai K (2018) Bacterial quorum sensing in symbiotic and pathogenic relationships with hosts. Biosci Biotechnol Biochem 82: 363-371.

Lorian V, Zak O, Suter J, Bruecher C (1985) Staphylococci, in vitro and in vivo. Diagn Microbiol Infect Dis 3: 433-444.

Persat A, Nadell CD, Kim MK, Ingremeau F, Siryaporn A, Drescher K, Wingreen NS, Bassler BL, Gitai Z, Stone HA (2015) The mechanical world of bacteria. Cell 161: 988-997.

Rahim K, Saleha S, Zhu X, Huo L, Basit A, Franco OL (2017) Bacterial contribution in chronicity of wounds. Microb Ecol 73: 710-721.

Sattler S, Kennedy-Lydon, T (2017) The immunology of cardiovascular homeostasis and pathology $1^{\text {st }}$ ed. Eds. Sattler and Kennedy-Lydon. Springer, 283 pp. 
Scales BS, Huffnagle GB (2013) The microbiome in wound repair and tissue fibrosis. J Pathol 229: 323331.

Tabor OB, Bosse MJ, Hudson MC, Greene KG, Nousiainen MT, Meyer RA, Sims SH, Kellam JF (1998) Does bacteremia occur during high pressure lavage of contaminated wounds? Clin Orthop Relat Res 347:117-121.

Zhao R, Liang H, Clarke E, Jackson C, Xue M (2016) Inflammation in chronic wounds. Int J Mol Sci 17: 2085. DOI: 10.3390/ijms17122085.

\section{Web reference}

1. https://qupath.github.io/

Editor's note: There were no questions from reviewers for this paper, therefore there is no Discussion with Reviewers section. The Guest Editor responsible for this paper was Willem-Jan Metsemakers. 